Series A

\author{
I. MATHEMATICA
}

491

\title{
EINIGE BEMERKUNGEN ÜBER ASYNCHRONE STOCHASTISCHE AUTOMATEN
}

\author{
vON
}

PETER H. STARKE

H E L S I N I 1971

S U O M A L A N E N T I D E A K T T M A

doi:10.5186/aasfm.1971.491 
Copyright (C) 1971 by

Academia Scientiarum Fennica

Am 9 Oktober 1970 vorgelegt von Arto SalomaA. 


\section{Einige Bemerkungen über asynchrone stochastische Automaten}

Die vorliegende Note ist aus einem Vortrag hervorgegangen, den ich auf dem von Prof. Dr. A. Salomaa veranstalteten Colloquium "Stochastische Automaten» (Turku, Mai 1970) gehalten habe. Es werden hier einige der Probleme gelöst, die von $\mathrm{H}$. Thiele und mir in unserer Arbeit [1] aufgeworfen wurden, die auch die Grundlage für den erwähnten Vortrag bildete. Aus Vollständigkeitsgründen gebe ich hier eine Übersicht über die wichtigsten Definitionen und Ergebnisse der Arbeit [1], versehen mit einige zusätzlichen Betrachtungen. Ferner führe ich einen Homomorphiebegriff ein, der es gestattet, synchrone stochastische Automaten homomorph auf asynchrone stochastische Automaten abzubilden.

Soweit nichts anderes verabredet ist, verwende ich dieselbe Terminologie wie in der Arbeit [2].

\section{Grundbegriffe}

In diesem Abschnitt geben wir die grundlegenden Definitionen und Ergebnisse aus der Theorie der asynchronen stochastischen Automaten an.

Definition 1.1. Ein Quadrupel $\mathfrak{C}=[X, Y, Z, H]$ heisst asynchroner stochastischer Automat, wenn $X, Y$ und $Z$ nichtleere Mengen sind und $H$ eine Funktion ist, die jedem Paar $[z, x]$ aus $Z \times X$ ein diskretes Wahrscheinlichkeitsmass $H[z, x]$ über $W(Y) \times Z$ zuordnet.

Interpretation. Das System $\mathfrak{C}$ arbeitet taktweise in einer diskreten Zeitskala $t=1,2,3, \ldots$ In jedem Takt $t$ befindet sich $\mathfrak{C}$ in genau einem Zustand $z$ aus der Zustandsmenge $Z$ von $\mathfrak{C}$, empfängt genau ein Eingabesignal $x$ aus dem Eingabealphabet $X$ von $\mathfrak{C}$ und sendet ein Wort $s$ aus der Wortmenge $W(Y)$ über dem Ausgabealphabet $Y$ von $\mathfrak{C}$ aus. Ist $z_{t}$ der Zustand von $\mathfrak{C}$ im Takt $t$ und $x_{t}$ das im Takt $t$ in $\mathfrak{C}$ eingegebene Signal, so ist $H\left[z_{t}, x_{t}\right]\left(s, z^{\prime}\right)$ die Wahrscheinlichkeit dafür, dass $\widetilde{c}$ im Takt $t$ das Wort $s$ ausgibt und im Takt $t+1$ im Zustand $z^{\prime}$ ist. Das im Takt $t$ ausgegebene Wort $s$ wird mit den in früheren Takten ausgebenen Wörtern »kommafrei» verkettet, d.h. ohne Trennzeichen an das bis zum Takt $t$ ausschliesslich produzierte Ausgabewort angefügt. 
A. I. 491

Bemerkung. Bei einem asynchronen stochastischen Automaten arbeiten also Eingabe und Ausgabe nicht synchronisiert. Auf Eingabe eines einzelnen Eingabesignals $x$ hin, d.h. in einem einzelnen »Eingabetakt», kann die Ausgabe ein ganzes Wort $s$ aus $W(Y)$ (unter Umständen auch das leere Wort $e$ ) produzieren, in diesem Fall arbeitet die Ausgabeeinheit also $l(s)$ Takte. Lediglich Eingabe und Zustandüberführung sind bei asynchronen stochastischen Automaten synchronisiert (auf jedes Eingabesignal hin wird genau einmal der Zustand gewechselt), daher ergeben sich bei der Betrachtung von Automaten ohne Ausgabe gegenüber der »synchronen» Theorie keine neuen Gesichtspunkte.

Wir definieren nun einige Eigenschaften von asynchronen stochastischen Automaten.

Definition 1.2. Es sei $\mathfrak{C}=[X, Y, Z, H]$ ein asynchroner stochastischer Automat.

1. Wir nennen $C$ synchron, wenn aus $H[z, x]\left(s, z^{\prime}\right)>0$ stets $s \in Y$, d.h. $l(s)=1$, folgt.

2. (5 heisst observabel, wenn es eine Funktion $\delta$ aus $Z \times X \times W(Y)$ in $Z$ derart gibt, dass $H[z, x]\left(s, z^{\prime}\right)$ genau dann nicht verschwindet, wenn $\delta$ für $[z, x, s]$ definiert und gleich $z^{\prime}$ ist.

3. 5 wird schwach-endlich genannt, wenn $X, Y$ und $Z$ endliche Mengen sind und endlich, wenn überdies die Menge

$$
W_{\mathscr{C}}=\left\{s \mid \text { 且 } z^{\prime} \mathrm{H} z^{\prime} \mathrm{H} x\left(z, z^{\prime} \in Z \wedge x \in X \wedge H[z, x]\left(s, z^{\prime}\right)>0\right)\right\}
$$

endlich ist.

4. Als zufällige Situation von $(5$ bezeichnen wir jedes diskrete Wahrscheinlichkeitsmass $\eta$ über $W(Y) \times Z$. Die zufällige Situation $\eta$ heisst endlich, wenn die Menge

$$
W_{\eta}=\{s \mid \eta(\{s\} \times Z)>0\}
$$

endlich ist.

Interpretation. Bei einem synchronen Automaten wird auf jedes Eingabesignal hin ein Wort der Länge 1 ausgegeben. Identifizieren wir wie üblich die Wörter der Länge 1 über $Y$ mit den Elementen $y$ von $Y$, so ergibt sich der übliche Begriff des stochastischen Automaten (vgl. z.B. [2]) als »synchroner» Spezialfall des Begriffs des asynchronen stochastischen Automaten.

Bei einem observablen Automaten kann aus der Kenntnis seines Zustandes $z$ im Takt $t$ und der Beobachtung des im Takt $t$ eingegebenen Signals $x$ einerseits und des im Takt $t$ ausgegebenen Wortes $s$ andererseits eindeutig auf seinen Zustand $\delta(z, x, s)=z^{\prime}$ im Takt $t+1$ geschlossen werden. 
Ist $\eta \in \Pi_{W(Y) \times Z}$ eine zufällige Situation von $\mathfrak{c}$, so interpretieren wir die Zahl $\eta(s, z)$ als Wahrscheinlichkeit dafür, dass der Automat 5 das Wort $s$ bereits produziert (auf sein Ausgabeband gedruckt) hat und sich im Zustand $z$ befindet.

Die Begriffe »zufälliger Zustand», »determinierter zufälligər Zustand» usw. werden wie in [2] benutzt. Der B griff der zufälligen Situation ist offenbar eine Verallgemeinerung des B griffs des zufälligen Zustandes. Ist $\pi \in \Pi_{Z}$ ein zufälliger Zustand von $\mathfrak{E}$, so definieren wir

$$
\eta_{\pi}(s, z)= \begin{cases}\pi(z), & \text { falls } s=e \\ 0, & \text { sonst. }\end{cases}
$$

Dabei ist $\eta_{\pi}$ eine zufällige Situation von 5 , die bei der üblichen Interpretation von $\pi$ genau dann vorliegt, wenn die Situation $\eta_{\pi}$ vorliegt. Umgekehrt gehört zu jeder Situation $\eta$ mit

$$
\eta(s, z)>0 \rightarrow s=e
$$

eindeutig der zufällige Zustand $\pi$ mit $\pi(z)=\eta(e, z)$. Überdies identifizieren wir die Zustände $z$ aus $Z$ mit den determinierten zufälligen $Z u$ ständen $\delta_{z}$ bzw. den determinierten zufälligen Situationen $\delta_{e, z}$.

Wir dehnen den Definitionsbereich von $H$ aus und definieren eine Funktion $V_{\mathfrak{c}}$, die das Verhalten von 5 bei der Verarbeitung von Wörtern $p$ aus $W(X)$ beschreibt:

Definition 1.3. Es sei $\mathfrak{E}=[X, Y, Z, H]$ ein asynchroner stochastischer Automat, $p=x_{1} \ldots x_{n} \in W(X), q \in W(Y), z_{1}, z_{n+1} \in Z, \eta \in \Pi_{W(Y) \times Z}$. Dann setzen wir

$H\left[z_{1}, p\right]\left(q, z_{n+1}\right)=\left\{\begin{array}{l}1, \text { falls } p=e(\text { d.h. } n=0), q=e, z_{1}=z_{n+1} ; \\ \sum_{\substack{s_{1}, \ldots s_{n}=q \\ s_{i} \in W(Y)}} \sum_{z_{2}, \ldots, z_{n} \in Z} \prod_{i=1}^{n} H\left[z_{i}, x_{i}\right]\left(s_{i}, z_{i+1}\right), \text { für } n>0 \\ 0, \text { sonst; }\end{array}\right.$

$$
\left.H[\eta, p]\left(q, z^{\prime}\right)=\sum_{s \in W(Y)} \sum_{z \in Z} \eta(s, z) H[z, p]\left(\frac{q}{s} \times\left\{z^{\prime}\right\}\right]\right),
$$

wobei $\frac{q}{s}=\{w \mid s w=q\}$ gesetzt ist, und

$$
V_{\mathfrak{c}}[\eta, p](q)=H[\eta, p](\{q\} \times Z) .
$$


Interpretation. In der Interpretation der Definition 1.1 haben wir vermerkt, dass die Ausgabe von $\mathfrak{c}$ kommafrei erfolgt, d.h. der Automat (5) setzt keine Trennzeichen zwischen die von ihm in aufeinanderfolgenden Takten ausgegebenen Wörter. Wenn 5 also zwei Takte gearbeitet hat und danach das Wort $w$ auf seinem Ausgabeband steht, dann kann man im allgemeinen nicht mehr feststellen, wie das Wort $w$ so in eine Verkettung $w=w_{0} w_{1} w_{2}$ zu zerlegen ist, dass $w_{0}$ das Wort ist, das bereits bei Bøginn der Arbeit von $\mathfrak{C}$ auf dem Ausgabeband gestanden hat, dass $w_{1}$ das im ersten und $w_{2}$ das im zweiten Takt dazugedruckte Wort ist. Wenn man das beachtet, so ist unmittelbar klar, dass $H[z, p]\left(q, z^{\prime}\right)=$ $H\left[\delta_{e, z}, p\right]\left(q, z^{\prime}\right) \quad$ (bzw. $\left.H[\eta, p]\left(q, z^{\prime}\right)\right)$ die Wahrscheinlichkeit dafür ist, dass nach Eirgabe des Wortes $p$ auf dem Ausgababand von $\mathfrak{c}$ das Wort $q$ steht und $\sqrt{5} \operatorname{im}$ Zustand $z^{\prime}$ ist, wenn $\widetilde{E}$ bəi B ginn seiner Arbeit, d.h. unmittelbar vor dem Eirg€ben von $p$, in der Situation $\delta_{e, z}$ (bzw. $\eta$ ) war. Damit ist $V_{\varepsilon}[\eta, p](q)$ die Wahrscheinlichkeit dafür, dass $\mathbb{E}$ ausg ahend von der Situation $\eta$ nach Eingabe von $p$ das Wort $q$ produziert hat.

Man zeigt nun (vgl. [1])

Satz 1.1. Es sei $\quad \mathcal{C}=[X, Y, Z, H]$ ein asynchroner stochastischer Automat, $\eta \in \Pi_{W(Y) \times Z}, p, r \in W(X), w \in W(Y), z^{\prime} \in Z$. Dann gilt

1. $H[\eta, e]=\eta$

2. $V_{1}[\eta, e](w)=\eta(\{w\} \times Z)$

3. $H[\eta, p] \in \Pi_{W(\mathrm{Y}) \times Z} \wedge V_{\mathrm{c}}[\eta, p] \in \Pi_{W(\mathrm{Y})}$

4. $H[\eta, p r]=H[H[\eta, p], r]$

5. $V_{\mathrm{e}}[\eta, p r]=V_{\mathrm{e}}[H[\eta, p], r]$.

\section{2. Äquivalenz}

In diesem Abschniot rekapitulieren wir die wichtigsten D finitionen und Erg€bnisse aus [1], die die Äquivalenz und schwache Äquivalenz von asynchronen stochastischen Automaten betreffen und lösen ein diesbezügliches Problem aus [1].

Definition 2.1. Es seien $\widetilde{C}=[X, Y, Z, H], \mathfrak{C}^{\prime}=\left[X, Y, Z^{\prime}, H^{\prime}\right]$ asynchrone stochastische Automaten mit dem gleichen Eingabealphabet und dem gleichen Ausgabealphabet.

1. Zufällige Situationen $\eta$ bzw. $\eta^{\prime}$ von $\subseteq$ bzw. $\complement^{\prime}$ werden äquivalent genannt (B॰z-ichnung: $\eta \sim \eta^{\prime}$ ), wenn für alle $p \in W(X)$ die Gleichung $V_{\mathrm{E}}[\eta, p]=V_{\mathfrak{E}}\left[\eta^{\prime}, p\right]$ gilt. 
2. $\widetilde{C}$ heisst schwach-äquivalent eingebettet in $\mathbb{C}^{\prime}$ (Bezeichnung: $\mathbb{C} \cong \mathbb{C}^{\prime}$ ), wenn zu jeder Situation $\eta$ von $\mathbb{E}$ eine äquivalente Situation $\eta^{\prime}$ von $5^{\prime}$ existiert.

3. Die Automaten $\mathfrak{C}, \mathbb{C}^{\prime}$ heissen schwach äquivalent $\left(\mathfrak{C} \approx \mathfrak{C}^{\prime}\right)$ wenn $\mathfrak{C} \cong \mathbb{C}^{\prime}$ und $\mathfrak{C}^{\prime} \cong \mathbb{E}$ ist.

Durch die Definition 2.1 ist auch definiert, wann ein Zustand $z$ von 5 äquivalent $\mathrm{zu}$ einem Zustand $z^{\prime}$ von $\widetilde{C}^{\prime}$ ist, da $z$ durch $\delta_{e, z}$ und $z^{\prime}$ durch $\delta_{e, z^{\prime}}$ beschrieben wird. Wie in [2] werden die Beziehungen "⿷ ist äquivalent eingebettet in $\mathfrak{C}^{\prime}\left(\mathfrak{C} \subseteq \mathfrak{C}^{\prime}\right) 》$ und $\gg \mathbb{C}^{\mathfrak{C}}$ ist äquivalent $\mathrm{zu} \mathfrak{C}^{\prime}$ $\left(\widetilde{C} \sim \mathfrak{C}^{\prime}\right) »$ definiert.

In dคr synchronen Theorie (vgl. [2], Teil III, Def. 2.1) sind die Relationen $\cong, \approx$ etwas anders definiert, dort heisst $\subseteq$ schwach äquivalent eingebettet in $C^{\prime}$, wenn gilt

$$
\mathrm{F} \pi\left(\pi \in \Pi_{Z} \rightarrow \boldsymbol{H} \pi^{\prime}\left(\pi^{\prime} \in \Pi_{Z^{\prime}} \wedge \pi \sim \pi^{\prime}\right)\right) .
$$

Man zeigt aber, dass beide Definitionen gleichwertig sind:

Satz 2.1. $ᄃ \underset{\complement}{\complement} \leftrightarrow \forall \pi\left(\pi \in \Pi_{Z} \rightarrow \mathbb{H} \pi^{\prime}\left(\pi^{\prime} \in \Pi_{Z^{\prime}} \wedge \pi \sim \pi^{\prime}\right)\right)$.

Daraus ergibt sich

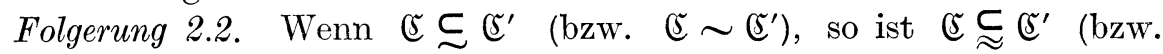
$\left(5 \approx c^{\prime}\right)$.

Es sei $\mathbb{E}=[X, Y, Z, H]$ ein asynchroner stochastischer Automat, $\eta$ eine zufällige Situation von $\mathfrak{5}, p \in W(X)$ und $q \in W(Y)$ mit $V_{\mathfrak{s}}[\eta, p](q)$ $>0$. Dann ist der zufällige Zustand $\mathfrak{E}[\eta, p, q]$ für $z \in Z$ definiert durch

$$
\mathfrak{C}[\eta, p, q](z)=\frac{H[\eta, p](q, z)}{V_{\mathfrak{G}}[\eta, p](q)} .
$$

Offenbar ist $\mathfrak{C}[\eta, p, q](z)$ die Wahrscheinlichkeit dafür, dass C im Zustand $z$ ist, wenn $\mathbb{C}$ ausgehend von der Situation $\eta$ bei Eingabe von $p$ das Wort $q$ produziert hat. Es gilt nun:

$$
\text { Folgerung 2.3. } \quad V_{\mathbb{E}}[\eta, p r](w)=\sum_{q: V_{\mathbb{E}[}[\eta, p](q)>0} V_{\mathbb{E}[\eta, p](q)} V_{\mathbb{E}}[\mathbb{E}[\eta, p, q], r]\left(\frac{w}{q}\right)
$$

Mit Hilfe von 2.3 kann man leicht zeigen:

Satz 2.4. Zu jedem asynchronen stochastischen Automaten $\mathbb{5}$ gibt es einen observablen asynchronen stochastischen Automaten $\mathfrak{C}^{*}$ mit $\mathfrak{C} \subseteq \mathfrak{C}^{*}$ und $\mathfrak{C} \approx \mathfrak{C}^{*}$.

Die Zustandsmenge $Z^{*}$ von $\mathbb{C}^{*}$ ist im allgemeinen unendlich, auch wenn 5 endlich ist. Zum Beweis wählt man $Z^{*}$ als die kleinste Menge 
von zufälligen Zuständen $\pi$ von $\mathfrak{C}$, welche die determinierten zufälligen Zustände $\delta_{z}$ enthält und abgeschlossen ist in bezug auf die Bildung der zufälligen Zustände $\subseteq[\pi, x, s]$ für $x \in X, s \in W(Y)$ mit $V_{\S}[\pi, x](s)>0$.

In der Arbeit [1] wurde folgendes Problem gestellt. Wenn $\mathfrak{C}=[X, Y, Z, H]$ ein asynchroner stochastischer Automat ist und $\eta$ eine Situation von $\mathfrak{C}$, ferner $p, p^{\prime} \in W(X), q, q^{\prime} \in W(Y)$ mit $V_{\mathbb{E}}[\eta, p](q)>0$ und $V_{\mathbb{E}}\left[\eta, p^{\prime}\right]\left(q^{\prime}\right)$ $>0$, sowie $\mathbb{E}[\eta, p, q] \sim \mathbb{E}\left[\eta, p^{\prime}, q^{\prime}\right]$, gilt dann $\mathbb{E}[\eta, p x, q s] \sim \mathbb{E}\left[\eta, p^{\prime} x, q^{\prime} s\right]$ für alle $x \in X, s \in W(Y)$ mit $V_{\mathbb{E}}[\mathbb{E}[\eta, p, q], x](s)>0$ ?

Wenn C synchron ist, so ist das tatsächlich der Fall, wie in [1] gezeigt wurde. Im allgemeinen gilt das jedoch nicht, wie das folgende Beispiel zeigt.

Wir betrachten den autonomen asynchronen stochastischen Automaten $\varsigma_{1}=\left[\{x\},\{a, b\},\{1,2, A, B, C, D\}, H_{1}\right]$, dessen Graph in der Abbildung 1 dargestellt ist. Dabei ist $\alpha$ eine reelle Zahl mit $0<\alpha<1$. Offenbar ist $\mathfrak{C}_{1}$ observabel und der Teilautomat $\mathfrak{C}_{2}$ von $\mathfrak{C}_{1}$ mit der Zustandsmenge $\{A, B, C, D\}$ ist sogar $Z$-determiniert.

Wir wählen nun $\eta$ mit

$$
\eta(s, z)=\left\{\begin{array}{l}
\frac{1}{2}, \text { für }[s, z] \in\{[a, 2],[a a, 1]\}, \\
0, \text { sonst, }
\end{array}\right.
$$

ferner $p=p^{\prime}=e, q=a$ und $q^{\prime}=a a$. Dabei ist

$$
V_{\mathbb{E}_{1}}[\eta, e](a)=V_{\mathfrak{E}_{1}}[\eta, e](a a)=\frac{1}{2}>0
$$

und

$$
\begin{aligned}
& \mathfrak{\Xi}_{1}[\eta, e, a](z)=\frac{H_{1}[\eta, e](a, z)}{V_{\mathbb{E}_{1}}[\eta, e](a)}=2 \eta(a, z)=\left\{\begin{array}{l}
1, \text { für } z=2, \\
0, \text { sonst }
\end{array}\right. \\
& \mathfrak{\complement}_{1}[\eta, e, a a](z)=2 \eta(a a, z)=\left\{\begin{array}{l}
1, \text { für } z=1 \\
0, \text { sonst }
\end{array}\right.
\end{aligned}
$$

Wir zeigen nun, dass die Zustände 1 und 2 von $\widetilde{G}_{1}$ äquivalent sind (d.h. $\left.\mathfrak{E}_{1}[\eta, e, a] \sim \mathfrak{E}_{1}[\eta, e, a a]\right)$. Es ist offenbar

$$
V_{\mathbb{E}_{1}}[1, x](s)=\left\{\begin{array}{l}
\frac{1}{2}, \text { für } s \in\{a, a a\} \\
0, \text { sonst }
\end{array}\right\}=V_{\mathbb{E}_{1}}[2, x](s)
$$

und für $n \geq 0, i \in\{1,2\}$

$$
V_{\mathbb{E}_{1}}\left[i, x^{n+1}\right](w)=V_{\mathbb{E}_{1}}\left[H_{1}[i, x], x^{n}\right](w) .
$$

Daher genügt es zu zeigen, dass die Situationen $\eta_{1}, \eta_{2}$ sowohl in $\mathfrak{S}_{1}$ als auch in $\mathfrak{夭}_{2}$ äquivalent sind, wobei 


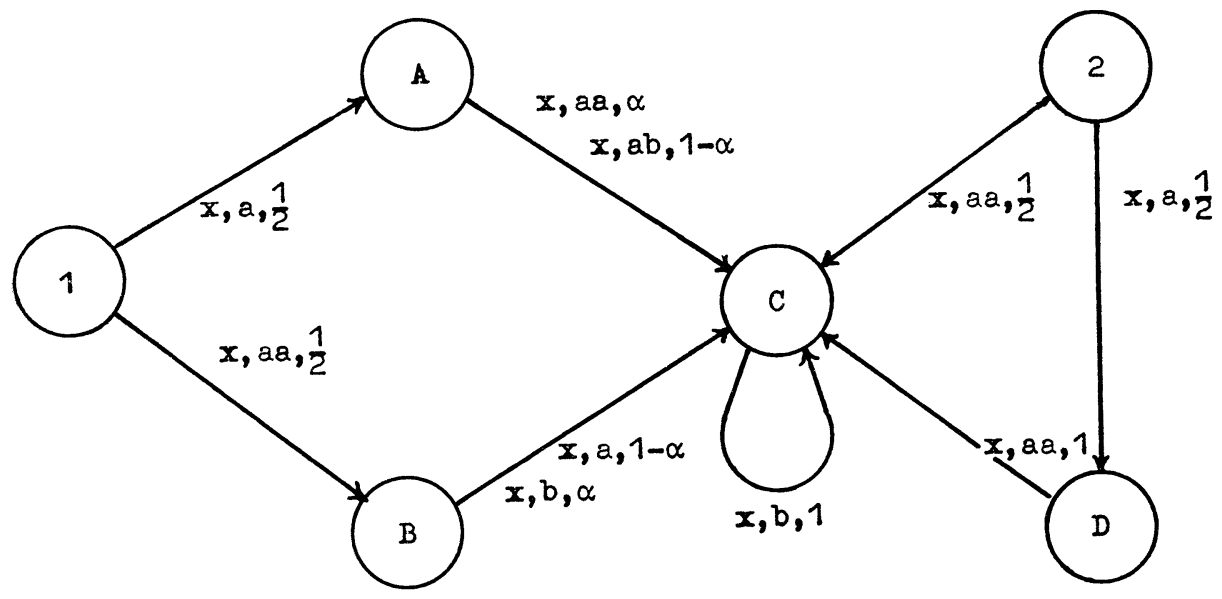

Abb. 1

$$
\begin{aligned}
& \eta_{1}(s, z)=\left\{\begin{array}{l}
\frac{1}{2}, \text { für }[s, z] \in\{[a, A],[a a, B]\}, \\
0, \text { sonst }
\end{array}\right. \\
& \eta_{2}(s, z)=\left\{\begin{array}{l}
\frac{1}{2}, \text { für }[s . z] \in\{[a, D],[a a, C]\}, \\
0, \text { sonst }
\end{array}\right.
\end{aligned}
$$

gesetzt ist. Wir beweisen das durch Induktion über $p$. Es ist klar, dass $V_{\mathfrak{E}_{2}}\left[\eta_{1}, e\right]=V_{\mathfrak{E}_{2}}\left[\eta_{2}, e\right]$ ist.

Schluss von $x$ auf $x p$ für $p \in(\{x\})$ :

$$
\begin{aligned}
& V_{\mathfrak{E}_{2}}\left[\eta_{1}, x p\right](w)=\frac{1}{2}\left(\alpha V_{\mathfrak{E}_{2}}[C, p]\left(\frac{w}{a a a}\right)+(1-\alpha) V_{\mathfrak{G}_{2}}[C, p]\left(\frac{w}{a a b}\right)\right)+ \\
& +\frac{1}{2}\left((1-\alpha) V_{\varepsilon_{2}}[C, p]\left(\frac{w}{a a a}\right)+\alpha V_{\mathbb{E}_{2}}[C, p]\left(\frac{w}{a a b}\right)\right) \\
& =\frac{1}{2} V_{\overleftarrow{G}_{2}}[C, p]\left(\frac{w}{a a a}\right)+\frac{1}{2} V_{E_{2}}[C, p]\left(\frac{w}{a a b}\right) \\
& =\frac{1}{2} \cdot \sum_{\substack{s \in W(Y) \\
z \in\{A, B, C, D,\}}} H_{2}[D, x](s, z) V_{\mathbb{E}_{2},}[z, p]\left(\frac{w}{a s}\right)+ \\
& +\frac{1}{2} \sum_{\substack{s \in W(Y) \\
z \in\{A, B, C, D\}}} H_{2}[C, x](s, z) V_{\mathbb{E}_{2}}[z, p]\left(\frac{w}{\text { aas }}\right) \\
& =V_{\mathfrak{G}_{2}}\left[\eta_{2}, x p\right](w) \text {. }
\end{aligned}
$$

Wir betrachten nun das Wort $s=a a$. Dabei ist 


$$
V_{\mathfrak{G}_{1}}\left[\mathfrak{C}_{1}[\eta, e, a a], x\right](s)=V_{\mathfrak{E}_{1}}[2, x](s)=\frac{1}{2}>0
$$

und (wie man leicht nachrechnet)

$$
\begin{aligned}
& \mathfrak{\subseteq}_{1}[\eta, x, \text { aaa }](z)=\left\{\begin{array}{l}
\frac{1}{2}, \text { für } z \in\{A, C\} \\
0, \text { sonst }
\end{array}\right. \\
& \mathfrak{夭}_{1}[\eta, x, \text { aaaa }](z)=\left\{\begin{array}{l}
1, \text { für } z=B, \\
0, \text { sonst, }
\end{array}\right.
\end{aligned}
$$

jedoch gilt

$$
V_{\mathfrak{E}_{1}}\left[\mathfrak{\complement}_{1}\left[\eta, x, a^{3}\right], x\right](a)=0 \neq 1-\alpha=V_{\mathfrak{G}_{1}}\left[\complement_{1}\left[\eta, x, a^{4}\right], x\right](a),
$$

d.h. $\mathfrak{C}_{1}\left[\eta, x, a^{3}\right], \mathfrak{\subseteq}_{1}\left[\eta, x, a^{4}\right]$ sind nicht äquivalent.

Das die Äquivalenz betreffende Hauptresultat von [1] ist der

Satz 2.5. Es sei $\mathfrak{C}=[X, Y, Z, H]$ ein endlicher asynchroner stochastischer Automat mit genau $n$ Zuständen. Dann gilt für zufällige Zustände $\pi, \pi^{\prime}$ von $\mathfrak{C}$ und endliche Situationen $\eta, \eta^{\prime}$ von $\mathfrak{E}$ :

1. $\pi \sim \pi^{\prime} \leftrightarrow \forall p\left(p \in W(X) \wedge l(p) \leq n-1 \rightarrow V_{\mathfrak{s}}[\pi, p]=V_{\mathbb{s}}\left[\pi^{\prime}, p\right]\right)$

2. $\eta \sim \eta^{\prime} \leftrightarrow \forall p\left(p \in W(X) \wedge l(p) \leq n \rightarrow V_{\varepsilon}[\eta, p]=V_{\mathbb{s}}\left[\eta^{\prime}, p\right]\right)$.

Die zweite Behauptung des Satzes wird mit Hilfe der ersten bewiesen. Dazu wählt man zwei Elemente $z_{0}, z_{0}^{\prime}$, die nicht in $Z$ liegen und definiert $Z^{\prime}=Z \cup\left\{z_{0}, z_{0}^{\prime}\right\}$, sowie

$$
H^{\prime}\left[z^{\prime}, x\right]\left(s, z^{\prime \prime}\right)=\left\{\begin{array}{l}
H\left[z^{\prime}, x\right]\left(s, z^{\prime \prime}\right), \text { falls } z^{\prime}, z^{\prime \prime} \in Z \\
\eta\left(s, z^{\prime \prime}\right), \text { falls } z^{\prime}=z_{0}, z^{\prime \prime} \in Z \\
\eta^{\prime}\left(s, z^{\prime \prime}\right), \text { falls } z^{\prime}=z_{0}^{\prime}, z^{\prime \prime} \in Z \\
0, \text { sonst. }
\end{array}\right.
$$

Dabei ist $\mathbb{C}^{\prime}=\left[X, Y, Z^{\prime}, H^{\prime}\right]$ ein endlicher asynchroner stochastischer Automat mit

$$
V_{\mathfrak{G}}[\eta, p]=V_{\mathfrak{G}}\left[z_{0}, x p\right], \quad V_{\mathfrak{G}}\left[\eta^{\prime}, p\right]=V_{\mathbb{E}}\left[z_{0}^{\prime}, x p\right]
$$

für alle $x \in X, p \in W(X)$. Wendet man nun 2.5.1. auf $\mathfrak{C}^{\prime}, z_{0}, z_{0}{ }^{\prime}$ an, so ergibt sich 2.5.2. ohne Schwierigkeiten.

Der Beweis für die erste Behauptung von Satz 2.5 wird analog zum Beweis des entsprechenden Satzes in der synchronen Theorje (Satz von CARLyle [3]) geführt. Man betrachtet die linearen Räume

$$
L_{i}=L\left(\left\{V_{\mathfrak{G}}[., p](q) \mid p \in W(X) \wedge q \in W(Y) \wedge l(p) \leq i\right\}\right),
$$

die von den auf $Z$ definierten reellwertigen Funktionen $V_{\mathfrak{c}}[., p](q)$ mit 
$l(p) \leq i$ aufgespannt werden, sowie das für $f \in L\left(\left\{\delta_{z} \mid z \in Z\right\}\right)$ definierte lineare Funktional $\varphi_{\pi, \pi^{\prime}}$ mit

$$
\varphi_{\pi, \pi^{\prime}}(f)=\sum_{z \in Z}\left(\pi(z)-\pi^{\prime}(z)\right) f(z) .
$$

Dabei ist $\pi \sim \pi^{\prime}$ genau dann, wenn $\varphi_{\pi, \pi^{\prime}}$ auf allen Räumen $L_{i}$ identisch verschwindet. Wegen $L_{i} \subseteq L_{i+1}$ und $\operatorname{Dim}\left(L_{i}\right) \leq n$ genügt es zu zeigen, dass aus $L_{i}=L_{i+1}$ stets $L_{i+1}=L_{i+2}$ folgt. Der Beweis dafür ist hier allerdings komplizierter als in der synchronen Theorie.

Der Satz 2.5 erscheint etwas überraschend unter dem Aspekt, dass GRIFFiths [4] gezeigt hat, dass die Äquivalenz von Zuständen endlicher asynchroner nicht-deterministischer Automaten eine unentscheidbare Relation ist. Dies zeigt einmal mehr, dass die Theorie der nicht-deterministischen Automaten keineswegs eine Simplifikation der Theorie der stochastischen Automaten ist.

Bei der äquivalenten bzw. schwach-äquivalenten Vereinfachung von asynchronen stochastischen Automaten treten keine neuen Schwierigkeiten auf. Insbesondere kann nachgewiesen werden, dass die Minimalität und die starke Reduziertheit entscheidbare Eigenschaften endlicher asynchroner stochastischer Automaten sind, wobei $\mathfrak{c}$ minimal genannt wird, wenn gilt

$$
\mathrm{V} z \operatorname{Vi}\left(z \in Z \wedge \pi \in \Pi_{Z} \wedge z \sim \pi \rightarrow \pi=\delta_{z}\right),
$$

und $\mathfrak{E}$ stark reduziert heisst, wenn gilt

$$
\forall \pi \forall \pi^{\prime}\left(\pi, \pi^{\prime} \in \Pi_{Z} \wedge \pi \sim \pi^{\prime} \rightarrow \pi=\pi^{\prime}\right) .
$$

Wir wollen $\mathbb{E}$ sehr stark reduziert nennen, wenn $\mathbb{E}$ die Bedingung

$$
\forall \eta \forall \eta^{\prime}\left(\eta, \eta^{\prime} \in \Pi_{\mathscr{W}(Y) \times Z} \wedge \eta \sim \eta \rightarrow \eta=\eta^{\prime}\right)
$$

erfüllt ist. Synchrone Automaten sind genau dann stark reduziert wenn sie sehr stark reduziert sind. In [1] wurde die Frage gestellt, ob das auch für asynchrone stochastische, Automaten gilt. Wir zeigen, dass das nicht der Fall ist.

Als Beispiel betrachten wir den Teilautomaten $\mathfrak{C}_{2}$ von $\mathfrak{C}_{1}$ (mit der Zustandsmenge $Z_{2}=\{A, B, C, D\}$ ). Wir haben schon gesehen, dass die Situationen $\eta_{1}, \eta_{2}$ von $\mathfrak{C}_{2}$ äquivalent, aber verschieden sind. Es genügt also zu zeigen, dass $\mathfrak{c}_{2}$ stark reduziert ist. Nun beweist man auf dieselbe Weise wie in der synchronen Theorie (vgl. [2]):

Satz 2.6. Ein asynchroner stochastischer Automat $\mathfrak{\complement}=[X, Y, Z, H]$ mit genau $n$ Zuständen ist genau dann stark reduziert, wenn 


$$
\operatorname{Dim}\left(L\left(\left\{V_{\mathfrak{c}}[., p](q) \mid p \in W(X) \wedge q \in W(Y)\right\}\right)\right)=n
$$

ist.

Die Funktionenmenge $\left\{V_{\mathfrak{E}_{2}}[., x](a), V_{\mathfrak{E}_{2}}[., x](a a), V_{\mathfrak{G}_{2}}[., x](a b), V_{\mathfrak{E}_{2}}[., x](b)\right\}$ ist linear unabhängig, also ist $\mathfrak{C}_{2}$ stark reduziert.

Nennt man $\mathbb{C}^{\prime}$ einen Sehr-Stark-Reduzierten von $\mathbb{C}$, wenn $\mathbb{C}^{\prime}$ sehr stark reduziert ist und schwach äquivalent zu $\mathfrak{C}$ ist, so ergibt sich, dass jeder Sehr-Stark-Reduzierte von $\subseteq$ ein Minimaler von $\subseteq$ ist. Da alle Minimalen desselben Automaten paarweise äquivalent sind und aus der Äquivalenz eines reduzierten Automaten $\mathfrak{C}^{\prime \prime}$ zu einem sehr stark reduzierten Automaten $C^{\prime}$ folgt, dass $C^{\prime \prime}$ schon selbst sehr stark reduziert ist, besitzt also ein asynchroner stochastischer Automat genau dann einen SehrStark-Reduzierten, wenn er einen Minimalen besitzt und alle seine Minimalen sehr stark reduziert sind.

\section{Stochastische Operatoren}

In diesem Abschnitt beschäftigen wir uns mit dem externen Verhalten von asynchronen stochastischen Automaten $\mathfrak{r}$, für die eine zufällige Situation als Anfangssituation festgelegt ist. Die dadurch bestimmte Eingabe-Ausgabe-Relation ist offenbar ein spezieller stochasticher Operator.

Definition 3.1. Es seien $X$ und $Y$ nichtleere Mengen.

1. Jede Funktion $\Phi: p \rightarrow \Phi_{p}$ von $W(X)$ in $\Pi_{W(Y)}$ ist ein stochastischer Operator über $[X, Y]$.

2. Ein stochastischer Operator $\Phi$ heisst synchron, wenn aus $\Phi_{p}(q)>0$ stets $l(p)=l(q)$ folgt.

3. Wir nennen den stochastischen Operator $\Phi$ über $[X, Y]$ in dem stochastischen Automaten $\mathfrak{C}=[X, Y, Z, H]$ durch die zufällige Situation $\eta$ erzeugt, wenn $\Phi_{p}=V_{\mathfrak{s}}[\eta, p]$ für alle $p \in W(X)$ ist.

4. Es sei $\Phi$ ein stochastischer Operator über $[X, Y]$. Eine Funktion $\Psi$, die jedem Paar $[p, q] \in W(X) \times W(Y)$ mit $\Phi_{p}(q)>0$ einen stochastischen Operator $\Psi[p, q]$ derart zuordnet, dass für alle $p, r \in W(X)$, $w \in W(Y)$

$$
\Phi_{p r}(w)=\sum_{q: \Phi_{p}(q)>0} \Phi_{p}(q) \cdot \Psi[p, q]_{r}\left(\frac{w}{q}\right)
$$

ist, heisst eine Zustandsfamilie von $\Phi$.

Satz 3.1. Ein stochastischer Operator $\Phi$ über $[X, Y]$ ist genau dann 
in einem asynchronen stochastischen Automaten (durch einen Zustand) erzeugbar, wenn $\Phi$ eine Zustandsfamilie besitzt (und $\Phi_{e}(e)=1$ ist).

Die Existenz wenigstens einer Zustandsfamilie zu jedem erzeugbaren stochastischen Operator ergibt sich sofort aus 2.3, man kann

$$
\Psi[p, q]_{r}(s)=V_{\mathbb{E}}[\mathfrak{C}[\eta, p, q], r](s)
$$

wählen, wenn $\Phi$ in $\mathfrak{E}$ durch $\eta$ erzeugt ist. Zum Beweis der Umkehrung setzt man

$$
Z=\left\{[p, q] \mid p \in W(X) \wedge q \in W(Y) \wedge \Phi_{p}(q)>0\right\}
$$

und

$H[[p, q], x](s, z)=\left\{\begin{array}{c}\Psi[p, q]_{x}(s), \text { falls } \Psi[p, q]_{x}(s)>0 \text { und } z=[p x, q s] ; \\ 0, \text { sonst, }\end{array}\right.$

und erhält einen asynchronen stochastischen Automaten $\mathfrak{S}=[X, Y, Z, H]$, in dem $\Phi$ durch die Situation $\eta$ mit

$$
\eta(s, z)=\left\{\begin{array}{l}
\Phi_{e}(s), \text { falls } \Phi_{e}(s)>0 \text { und } z=[e, s] \\
0, \text { sonst }
\end{array}\right.
$$

erzeugt wird. Bei dieser Konstruktion ist die Menge $Z$ stets unendlich, man fragt sich daher, ob es nicht möglich ist, die Abbildungen $\Psi[p, q]$ selbst als Zustände von $\mathfrak{C}$ zu verwenden. Das ist ohne weiteres nur möglich, wenn aus $\Psi[p, q]=\Psi\left[p^{\prime}, q^{\prime}\right]$ und $\Psi[p, q]_{x}(s)>0$ stets $\Psi[p x, q s]=$ $=\Psi\left[p^{\prime} x, q^{\prime} s\right]$ folgt.

Wir werden unten sehen, dass es erzeugbare stochastische Operatoren gibt (nämlich die erzeugbaren separablen stochastischen Operatoren), bei denen jede Zustandsfamilie diese Eigenschaft hat. Andererseits hat die Zustandsfamilie $\Psi^{\circ}$ des in dem oben betrachteten stochastischen Automaten $\widetilde{r}_{1}$ durch $\eta$ erzeugten stochastischen Operators $\Phi$ mit

$$
\Psi^{\circ}[p, q](s)=V_{\overleftarrow{E}_{1}}\left[\complement_{1}[\eta, p, q], r\right](a s)
$$

diese Eigenschaft nicht, denn es ist

$$
\mathfrak{E}_{1}[\eta, e, a] \sim \mathfrak{E}_{1}[\eta, e, a a], \text { also } \Psi^{\circ}[e, a]=\Psi^{\circ}[e, a a],
$$

aber $\mathfrak{S}_{1}[\eta, x, a a a] \sim \mathfrak{S}_{1}[\eta, x, a a a a]$, also $\Psi^{\circ}[x, a a a]+\Psi^{\circ}[x$, aaaa $]$, obwohl $\Psi^{\circ}[e, a]_{x}(a a)=\frac{1}{2}>0$ ist. Indessen ist auch $\Psi^{1}$ mit

$$
\begin{aligned}
& \Psi^{1}[p, q]_{r}(s)=V_{\mathfrak{E}_{1}}\left[\mathfrak{\complement}_{1}[\eta, p, q], r\right](s), \text { für } p \neq e \\
& \Psi^{1}[e, a]_{e}(w)=\Psi^{1}[e, a a]_{e}(w)=\left\{\begin{array}{l}
1, \text { falls } w=e, \\
0, \text { sonst }
\end{array}\right.
\end{aligned}
$$




$$
\begin{aligned}
\Psi^{1}[e, a]_{x}(w) & =\left\{\begin{array}{l}
1, \text { falls } w=a a \\
0, \text { sonst }
\end{array}\right. \\
\Psi^{1}[e, a a]_{x}(w) & =\left\{\begin{array}{l}
\frac{1}{2}, \text { falls } w \in\{e, a a\} \\
0, \text { sonst }
\end{array}\right. \\
\Psi^{1}[e, a]_{x^{n+2}}(w) & =\Psi^{1}[e, a a]_{x^{n+2}}(w)=\left\{\begin{array}{l}
\frac{1}{2}, \text { für } w \in\left\{a^{3} b^{n}, a^{2} b^{n+1}\right\} \\
0, \text { sonst },
\end{array}\right.
\end{aligned}
$$

eine Zustandsfamilie für $\Phi$. Ist nun $\Psi^{1}[p, q]=\Psi^{1}\left[p^{\prime}, q^{\prime}\right]$ und $[p, q] \neq$ $\neq\left[p^{\prime}, q^{\prime}\right]$, so ist nicht $p=p^{\prime}=e$ oder $p=p^{\prime}=x$. Ist $p=x^{n}$, $p^{\prime}=x^{m}, n, m \geq 2, \quad$ so $\quad$ ist $\quad \mathfrak{S}_{1}[\eta, p, q]=\mathfrak{E}_{1}\left[\eta, p^{\prime}, q^{\prime}\right]=\delta_{C} \quad$ und einzig für $s=b$ ist $\Psi^{1}[p, q]_{x}(s)>0$. Dabei ist $\mathfrak{c}_{1}[\eta, p x, q b]=\mathfrak{c}_{1}\left[\eta, p^{\prime} x, q^{\prime} b\right]=$ $=\delta_{C}$, also $\Psi^{1}[p x, q s]=\Psi^{1}\left[p^{\prime} x, q^{\prime} s\right]$. Der Fall $p=e, p^{\prime}=x^{n+2}(n \geq 0)$ kann nicht eintreten wegen $\Psi^{1}[e, q]_{x}(b)=0 \neq 1=\Psi^{1}\left[x^{n+2}, q^{\prime}\right]_{x}(b)$. Es bleibt also nur noch der Fall $p=e, p^{\prime}=x$ zu untersuchen. Dabei ist $q \in\{a, a a\}, q^{\prime} \in\{a a, a a a, a a a a\}$. Bei $\Psi^{1}[e, q]=\Psi^{1}\left[x, q^{\prime}\right]$ ergäbe sich ,

$$
\begin{gathered}
\frac{1}{2}=\Psi^{1}[e, q]_{x x}(a a a)=\Psi^{1}\left[x, q^{\prime}\right]_{x x}(a a a) \\
\Phi_{x x x}\left(q^{\prime} a a a\right) \geq \Phi_{x}\left(q^{\prime}\right) \Psi^{1}\left[x, q^{\prime}\right]_{x x}(a a a)=\frac{1}{2} \Phi_{x}\left(q^{\prime}\right)>0,
\end{gathered}
$$

im Widerspruch zu $\Phi_{x x x}(w a)=0$ für alle $w \in W(Y)$. Der stochastische Operator $\Phi$ bildet also kein Gegenbeispiel für die Vermutung, dass jeder erzeugbare stochastische Operator eine Zustandsfamilie $\Psi$ besitzt, bei der aus $\Psi[p, q]=\Psi\left[p^{\prime}, q^{\prime}\right] \quad$ und $\Psi[p, q]_{x}(s)>0$ stets $\Psi[p x, q s]=$ $=\Psi\left[p^{\prime} x, q^{\prime} s\right]$ folgt.

Ist $\Phi$ ein erzeugbarer stochastischer Operator, so besitzt $\Phi$ eine Zustandsfamilie $\Psi$, deren Elemente $\Psi[p, q]$ sämtlich erzeugbare stochastische Operatoren sind. In [1] wurde die Frage gestellt, ob das sogar bei jeder Zustandsfamilie von $\Phi$ der Fall ist. Die Antwort auf diese Frage ist negativ.

Als Beispiel betrachten wir den im Automaten $\mathfrak{r}_{3}=[\{x\},\{a\},\{\mathrm{I}, \mathrm{II}$, III $\left.\}, H_{3}\right]$, dessen Graph die Abbildung 2 zeigt, durch den Zustand I erzeugten stochastischen Operator $\Phi^{\prime}$ über $[\{x\},\{a\}]$. Dabei ist

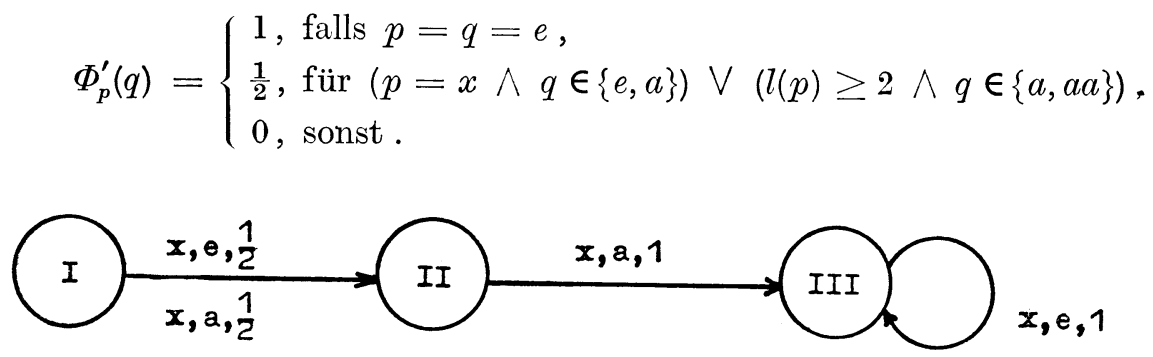

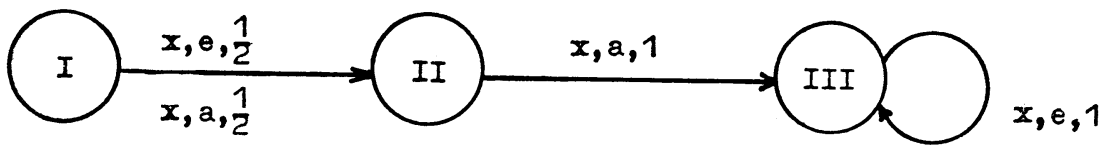

Abb. 2 
Wie betrachten die Funktion $\Psi^{\prime}$ mit

$$
\begin{aligned}
& \Psi^{\prime}[e, e]= \Phi^{\prime} \\
& \Psi^{\prime}[x, e]_{x^{n}}(w)=\left\{\begin{array}{l}
1, \text { für } n=0, w=e, \\
\frac{1}{4}, \text { für } n=1, w=a, \\
\frac{3}{4}, \text { für } n=1, w=a a, \\
\frac{1}{2}, \text { für } n \geq 2, w \in\{a, a a\}, \\
0, \text { sonst } ;
\end{array}\right. \\
& \Psi^{\prime}[x, a]_{x^{n}}(w)=\left\{\begin{array}{l}
1, \text { für } n=0, w=e, \\
\frac{3}{4}, \text { für } n=1, w=e, \\
\frac{1}{4}, \text { für } n=1, w=a, \\
\frac{1}{2}, \text { für } n \geq 2, w \in\{e, a\} \\
0, \text { sonst } ;
\end{array}\right. \\
& \Psi^{\prime}\left[x^{n+2}, a\right]_{x^{m}}(w)=\left\{\begin{array}{l}
1, \text { für } w=e \\
0, \text { sonst }
\end{array}\right\}=\Psi^{\prime}\left[x^{n+2}, a a\right]_{x^{m}}(w) .
\end{aligned}
$$

Offensichtlich ist $\Psi^{\prime}$ eine eindeutige Abbildung, die jedem $[p, q]$ mit $\Phi_{p}^{\prime}(q)>0$ einen stochastischen Operator über $[\{x\},\{a\}]$ zuordnet. Wir behaupten, dass $\Psi^{\prime}$ sogar eine Zustandsfamilie von $\Phi^{\prime}$ ist, d.h.

$$
\Phi_{x^{n} x^{m}}^{\prime}(w)=\sum_{q: \Phi_{x^{n}}^{\prime}(q)>0} \Phi_{x^{n}}^{\prime}(q) \Psi^{\prime}\left[x^{n}, q\right]_{x^{m}}\left(\frac{w}{q}\right)
$$

gilt für alle $n, m \geq 0, w \in W(Y)$. Diese Gleichung ist trivial für $n=0$ (wegen $\Phi_{o}^{\prime}(e)=1$ ) und $n \geq 2$. Bei $n=1$ ist

$$
\begin{aligned}
& \sum_{q: d_{x}^{\prime}(q)>0} \Phi_{x}^{\prime}(q) \Psi^{\prime}[x, q]_{x^{m}}\left(\frac{q}{w}\right)=\frac{1}{2}\left(\Psi^{\prime}[x, e]_{x^{m}}(w)+\Psi^{\prime}[x, a]_{x^{m}}\left(\frac{w}{a}\right)\right) \\
& =\left\{\begin{array}{l}
\frac{1}{2}, \text { für }(m=0 \wedge w \in\{e, a\}) \vee(m \geq 1 \wedge w \in\{a, a a\}) \\
0, \text { sonst }, \\
=\Phi_{x^{m+1}}^{\prime}(w),
\end{array}\right.
\end{aligned}
$$

was wir zeigen wollten. Der stochastische Operator $\Psi^{\prime}[x, e]$ ist aber nicht erzeugbar, denn es gilt

$$
\frac{3}{4}=\Psi^{\prime}[x, e]_{x}(a a)>\sum_{s \in W(Y)} \Psi^{\prime}[x, e]_{x x}(a a s)=\frac{1}{2}
$$

im Widerspruch zur vierten Behauptung des unten angegebenen Satzes 3.2, der in [1] bewiesen ist. Der auf der Basis von $\Psi^{\prime}$ nach Satz 3.1 konstruierte Automat zur Erzeugung von $\Phi$ hat den in der Abbildung 3 angedeuteten Graphen. 


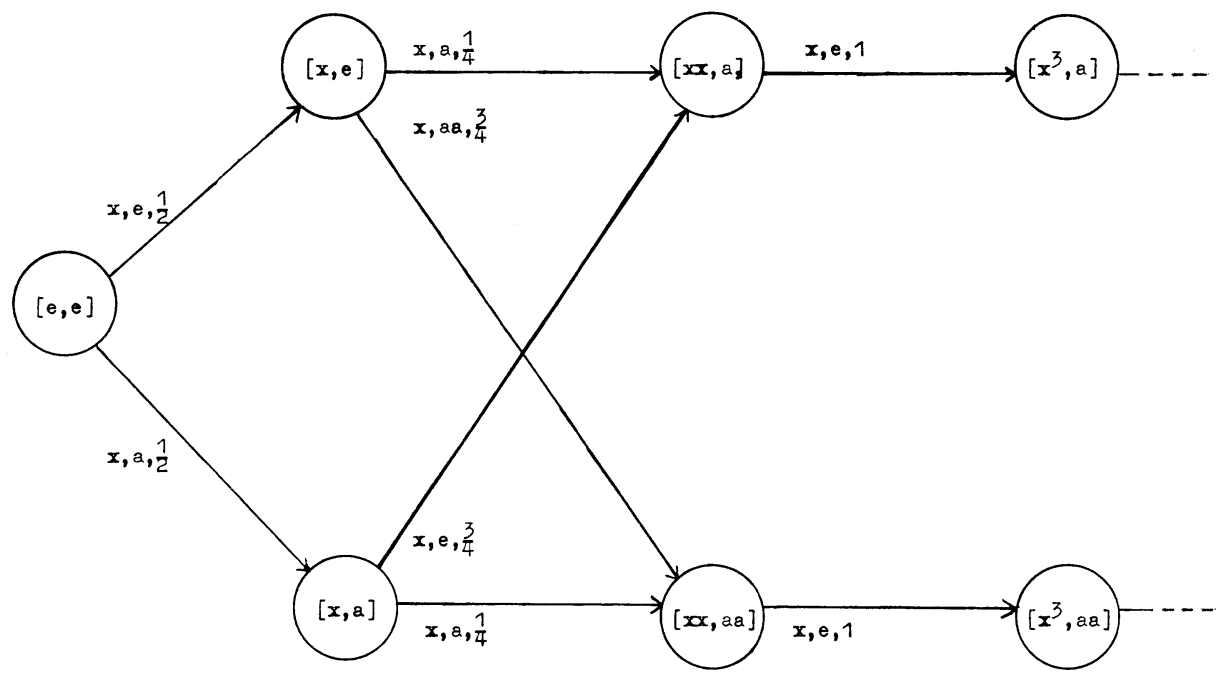

Abb. 3

Satz 3.2. Ist $\Psi$ eine Zustandsfamilie des stochastischen Operators $\Phi$ über $[X, Y]$, so gilt

1. $\Phi_{e}(e)=1 \rightarrow \Psi[e, e]=\Phi$.

2. $\forall p \forall q\left(p \in W(X) \wedge q \in W(Y) \wedge \Phi_{p}(q)>0 \rightarrow \Psi[p, q]_{e}(e)=1\right)$

3. $\forall p \forall r \forall w\left(p, r \in W(X) \wedge w \in W(Y) \rightarrow \Phi_{p r}(w) \leq \sum_{q: \frac{q}{w} \neq \varnothing} \Phi_{p}(q)\right)$

4. $\forall p \forall q \forall r\left(p, r \in W(X) \wedge q \in W(Y) \rightarrow \Phi_{p}(q) \leq \sum_{s \in W(Y)} \Phi_{p r}(q s)\right)$

Wir bemerken noch, dass in [1] durch ein Beispiel belegt ist, dass die Bedingungen 3.2.3 und 3.2.4 nicht hinreichend für die Erzeugbarkeit von $\Phi$ sind.

Wir haben schon gesehen, dass ein erzeugbarer stochastischer Operator im allgemeinen mehr als eine Zustandsfamilie besitzt. Es gilt sogar

Satz 3.3. Ist $\mathfrak{M}$ eine (nichtleere) höchstens abzählbar unendliche Menge von Zustandsfamilien von $\Phi$ und $\varphi$ eine Funktion von $\mathfrak{M}$ in das reelle Interval $[0,1]$ mit $\sum_{\Psi \in \mathfrak{M}} \varphi(\Psi)=1$, so ist $\Psi^{\varphi}$ mit

$$
\begin{array}{r}
\Psi^{\varphi}[p, q](s)=\sum_{\Psi \in \mathfrak{M}} \varphi(\Psi) \Psi[p, q]_{r}(s) \text { (für } p, r \in W(X), q, s \in W(Y) \\
\text { mit } \left.\Phi_{p}(q)>0\right)
\end{array}
$$

eine Zustandsfamilie von $\Phi$.

Beweis. Es ist klar, dass $\Psi^{\varphi}[p, q]$ stets ein stochastischer Operator über $[X, Y]$ ist und dass gilt 


$$
\begin{aligned}
\sum_{q: \Phi_{p}(q)>0} \Phi_{p}(q) \Psi^{\varphi}[p, q]_{r}\left(\frac{w}{q}\right) & =\sum_{q: \Phi_{p}(q)>0} \Phi_{p}(q) \sum_{\psi^{\prime} \in M} \varphi(\Psi) \Psi[p, q]_{r}\left(\frac{w}{q}\right) \\
& =\sum_{\Psi \in M M} \varphi(\Psi) \sum_{q: \Phi_{p}(q)>0} \Phi_{p}(q) \Psi[p, q]_{r}\left(\frac{w}{q}\right) \\
& =\sum_{\Psi \in M ?} \varphi(\Psi) \Phi_{p r}(w)=\Phi_{p r}(w) .
\end{aligned}
$$

Aus dem Satz 2.3 ergibt sich, dass jeder stochastische Operator, der wenigstens zwei (verschiedene) Zustandsfamilien besitzt, sogar kontinuierlich viele Zustandsfamilien hat. Wir suchen daher nach einer hinreichenden Bedingung dafür, dass ein erzeugbarer stochastischer Operator genau eine Zustandsfamilie besitzt (wobei dann jedes Element $\Psi[p, q]$ dieser Zustandsfamilie notwendig ein erzeugbarer stochastischer Operator ist und zwar wird $\Psi[p, q]$ in dem beim Beweis von 3.1 konstruierten Automaten vom Zustand $[p, q]$ erzeugt).

Definition 3.2. Ein stochastischer Operator $\Phi$ über $[X, Y]$ hejsst separabel, wenn zu jedem $p, r \in W(X)$ und $w \in W(Y)$ mit $\Phi_{p r}(w)>0$ genau ein Anfangsstück $q$ von $w$ mit $\Phi_{p}(q)>0$ existiert.

Natürlich ist jeder synchrone sequentielle stochastische Operator separabel. Man zeigt nun

Satz 3.4. Jeder separabel stochastische Operator besitzt höchstens eine Zustandsfamilie. Ist $\Phi$ separabel und $\Psi$ eine Zustandsfamilie von $\Phi$, $\Phi$, so ist (für $p, r \in W(X), q, s \in W(Y)$ mit $\Phi_{p}(q)>0$ )

$$
\Psi[p, q]_{r}(s)=\frac{\Phi_{p r}(q s)}{\Phi_{p}(q)} .
$$

Selbstverständlich existieren separable stochastische Operatoren, die keine Zustandsfamilie besitzen, die also nicht erzeugbar sind.

Folgerung 3.5. Wenn $\Phi$ ein separabler stochastischer Operator ist, der eine Zustandsfamilie besitzt, so gilt

$$
\forall p \forall q \forall r\left(p, r \in W(X) \wedge q \in W(Y) \wedge \Phi_{p}(q)>0 \rightarrow \sum_{s \in W(Y)} \Phi_{p r}(q s)=\Phi_{p}(q)\right)
$$

Satz 3.6. Es sei $\Phi$ ein beliebiger stochastischer Operator über $[X, Y]$. 1. Wenn $\Phi$ erzeugbar ist, so ist $\Phi$ genau dann separabel, wenn $\Phi$ die Behauptung von 3.5. erfüllt.

2. Wenn $\Phi$ separabel ist, so ist $\Phi$ genau dann erzeugbar, wenn $\Phi$ die Behauptung von 3.5. erfüllt.

3. Wenn $\Phi$ die Behauptung von 3.5 erfüllt, so ist $\Phi$ genau dann erzeugbar, wenn $\Phi$ separabel ist. 
Man überlegt sich auch ohne Schwierigkeiten, dass alle Elemente $\Psi[p, q]$ der Zustandsfamilie $\Psi$ eines separablen und erzeugbaren stochastischen Operators selbst separabel sind.

Wir schliessen diesen Abschnitt mit einer Bemerkung über die Realisierbarkeit von stochastischen Operatoren. In [2] wird eine Wortfunktion $\varphi \quad \varepsilon$-realisierbar (realisierbar mit der Endmarkierung $\varepsilon$ ) genannt, wenn es einen determinierten (asynchronen) Automaten $[X, Y, Z, \delta, \lambda]$ und einen Zustand $z \in Z$ derart gibt, dass $\varphi(p)=\lambda(z, p \varepsilon)$ für alle $p \in W(X)$ gilt. In Analogie dazu definieren wir (für ein beliebig fixiertes Element $\varepsilon \notin X)$ :

Definition 3.3. Der stochastische Operator $\Phi$ über $[X, Y]$ heisst durch die Situation $\eta$ in den asynchronen stochastischen Automaten $\mathfrak{C}=[X \cup\{\varepsilon\}, Y, Z, H]$ realisiert, wenn $\Phi_{p}=V_{\mathfrak{c}}[\eta, p \varepsilon]$ für alle $p \in W(X)$ ist.

Satz 3.7. Jeder stochastische Operator kann in einem Z-determinierten asynchronen stochastischen Automaten durch einen Zustand realisiert werden.

Zum Beweis setzt man $Z=W(X)$ und für $p, z^{\prime} \in Z, x \in X, s \in W(Y)$

$$
\begin{aligned}
& H[p, x]\left(s, z^{\prime}\right)=\left\{\begin{array}{l}
1, \text { falls } s=e \text { und } z^{\prime}=p x, \\
0, \text { sonst }
\end{array}\right. \\
& H[p, \varepsilon]\left(s, z^{\prime}\right)=\left\{\begin{array}{l}
\Phi_{p}(s), \text { falls } z^{\prime}=e, \\
0, \text { sonst } .
\end{array}\right.
\end{aligned}
$$

Offensichtlich ist hierbei $\widetilde{S}=[X \cup\{\varepsilon\}, Y, W(X), H]$ ein $Z$-determinierter asynchroner stochastischer Automat, in dem $\Phi$ vom Zustand $e$ realisiert wird.

Das Problem, welche stochastischen Operatoren in endlichen asynchronen stochastischen Automaten realisiert werden können, bleibt offen.

\section{Homomorphie}

Wir definieren hier einen Homomorphiebegriff für asynchrone stochastische Automaten, der etwas allgemeiner ist, als der für synchrone Automaten brkannte Homomorphiebegriff. Diese Verallgemeinerung betrifft die Abbildung $v$, die den Ausgabewörtern des betrachteten Automaten jene seines homomorphen Bildes zuordnet. Bei dem hier argrgebenen Homomorphiebegriff ist das homomorphe Bild eines synchronen Automaten nicht notwendig ein synchroner Automat.

Definition 4.1. Es seien $\quad \widetilde{C}=[X, Y, Z, H], \quad \mathbb{C}^{\prime}=\left[X^{\prime}, Y^{\prime}, Z^{\prime}, H^{\prime}\right]$ asynchrone stochastische Automaten. Ein Tripel $\chi=[\xi, v, \zeta]$ von ein- 
deutigen Abbildungen $\xi$ von $X$ auf $X^{\prime}, \nu$ von $Y$ in $W\left(Y^{\prime}\right)$ und $\zeta$ von $Z$ auf $Z^{\prime}$ heisst Homomorphismus von $\mathfrak{C}$ auf $\mathfrak{C}^{\prime}$, wenn für alle $z \in Z, x \in X, s^{\prime} \in W\left(Y^{\prime}\right), z^{\prime} \in Z^{\prime}$ gilt

$$
H^{\prime}[\zeta(z), \xi(x)]\left(s^{\prime}, z^{\prime}\right)=\sum_{z^{*}: \zeta\left(z^{*}\right)=z^{\prime}} \sum_{s: v(s)=s^{\prime}} H[z, x]\left(s, z^{*}\right),
$$

wobei $v$ durch die induktive Definition $v(e)=e, v(q y)=v(q) v(y)$ auf die Menge $W(Y)$ ausgedehnt ist. Wir sprechen von einem Z-Homomorphismus $\chi$ von $\subseteq$ auf $\complement^{\prime}$, wenn $X=X^{\prime}, Y=Y^{\prime}, \xi=I_{X}$ (die identische Abbildung von $X$ auf sich) und $v=I_{Y}$ ist.

Satz 4.1. Ist $[\xi, v, \zeta]$ ein Homomorphismus von $\subseteq=[X, Y, Z, H]$ auf $\mathbb{C}^{\prime}=\left[X^{\prime}, Y^{\prime}, Z^{\prime}, H^{\prime}\right)$, so gilt für alle $z \in Z, p \in W(X), w^{\prime} \in W\left(Y^{\prime}\right)$

$$
V_{\mathbb{G}^{\prime}}[\zeta(z), \xi(p)]\left(w^{\prime}\right)=\sum_{w: v(w)=w^{\prime}} V_{\mathbb{E}}[z, p](w) .
$$

Wir führen den Beweis durch Induktion über $p$. Im Anfangsschritt sei $p=e$. Dann ist $\xi(p)=e$ und die linke Seite der behaupteten Gleichung verschwindet genau dann nicht, wenn $w^{\prime}=e$ ist. Anderseits ist auch $V_{\varepsilon}[z, e](w)>0$ genau dann, wenn $w=e$ ist. Wegen $v(e)=e$ ist die B hauptung also für $p=e$ erfüllt. Wir schliessen nun von $p$ auf $x p$ für $x \in X$. Es ist

$$
\begin{aligned}
V_{\mathbb{E}^{\prime}}[\zeta(z), \xi(x p)]\left(w^{\prime}\right)=\sum_{s^{\prime} \in W\left(Y^{\prime}\right)} \sum_{z^{\prime} \in Z^{\prime}} H^{\prime}[\zeta(z), \xi(x)]\left(s^{\prime}, z^{\prime}\right) \cdot V_{\mathbb{E}^{\prime}}\left[z^{\prime}, \xi(p)\right]\left(\frac{w^{\prime}}{s^{\prime}}\right) \\
=\sum_{s^{\prime} \in W\left(Y^{\prime}\right)} \sum_{z^{*} \in Z} \sum_{z^{*}: \xi\left(z^{*}\right)=z^{\prime}} \sum_{s: v(s)=s^{\prime}} H[z, x]\left(s, z^{*}\right) \cdot V_{\mathbb{E}^{\prime}}\left[\zeta\left(z^{*}\right), \xi(p)\right]\left(\frac{w^{\prime}}{s^{\prime}}\right) \\
=\sum_{s^{\prime} \in W\left(Y^{\prime}\right)} \sum_{z^{*} \in Z} \sum_{s: v(s)=s^{\prime}} H[z, x]\left(s, z^{*}\right) \sum_{q: s^{\prime} v(q)=w^{\prime}} V_{\mathbb{E}[}\left[z^{*}, p\right](q) \\
=\sum_{s^{\prime}, q: s q^{\prime}=w^{\prime}} \sum_{s: v(s)=s^{\prime}} \sum_{q: v(q)=q^{\prime}} \sum_{z^{*} \in Z} H[z, x]\left(s, z^{*}\right) \cdot V_{\mathbb{E}}\left[z^{*}, p\right](q) \\
=\sum_{w: v(w)=w^{\prime}} \sum_{s \in W(Y)} \sum_{z^{*} \in Z} H[z, x]\left(s, z^{*}\right) V_{\Xi\left[z^{*}, p\right]}\left(\frac{w}{s}\right) \\
=\sum_{w: v(w)=w^{\prime}} V_{\mathbb{E}}[z, x p](w),
\end{aligned}
$$

was zu beweisen war.

Auf dieselbe Weise wie 4.1 beweist man unter Benutzung von 4.1 den

Satz 4.2. Es sei $\chi=[\xi, v, \zeta]$ ein Homomorphismus von $\mathfrak{C}$ auf $\mathfrak{C}^{\prime}$ und für $\eta \in \Pi_{W(Y) \times z}$ sei

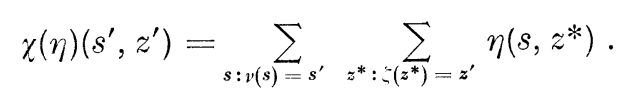


Dann gilt für alle $\eta \in \Pi_{W(Y) \times Z}, p \in W(X), w^{\prime} \in W\left(Y^{\prime}\right)$

$$
V_{\mathbb{E}}[\chi(\eta), \xi(p)]\left(w^{\prime}\right)=\sum_{w: \nu(w)=w^{\prime}} V_{\mathbb{E}}[\eta, p](w) .
$$

Folgerung 4.3. Ist $\chi=\left[I_{X}, I_{X}, \zeta\right]$ ein $Z$-Homomorphismus von $\mathfrak{E}=[X, Y, Z, H]$ auf $\mathbb{C}^{\prime}=\left[X, Y, Z^{\prime}, H^{\prime}\right], \quad$ so ist $\eta \sim \chi(\eta)$ für alle $\eta \in \Pi_{W(Y) \times Z}, z \sim \zeta(z)$ für alle $z \in Z$ und $\mathfrak{C} \sim \mathfrak{S}^{\prime}$.

Satz 4.4. Jeder asynchrone stochastische Automat ist homomorphes Bild eines synchronen stochastischen Automaten bei einem Homomorphismus, der nur aus eineindeutigen Abbildungen besteht.

Zum Beweis betrachtet man zu gegebenem asynchronen stochastischen Automaten $\mathfrak{E}=[X, Y, Z, H]$ den Automaten $\mathfrak{C}^{*}=\left[X, W_{\mathfrak{E}}, Z, H\right]$ und den Homomorphismus $\left[I_{X}, I_{W_{\tilde{E}}}, I_{Z}\right]$ (man beachte, dass z.B. das leere Wort $e$, wenn es in $W_{\mathfrak{G}}$ liegt, ein Ausgabebuchstabe von (5* ist).

Wenn der Satz 4.4. auch trivial ist, so hat er doch die interessante Folgerung, dass ein stochastischer Operator genau dann in einem $Z$-endlichen asynchronen stochastischen Automaten durch einen Zustand erzeugt werden kann, wenn er das $Y$-homomorphe Bild eines regulären (synchronen sequentiellen) stochastischen Operators ist.

Sektion Mathematik

der Humboldt-Universität zu Berlin

DDR-108 Berlin, Unter den Linden 6 


\section{Literatur}

[1] Starke, P. H., Thiele, H.: On asynchronous stochastic automata. Information and Control 17 (1970) 3, 265-293.

[2] -»- Abstrakte Automaten - VEB Deutscher Verlag der Wissenschaften, Berlin 1969 .

[3] Carlyle, J. W.: Reduced forms for stochastic sequential machines - J. Math. Anal. and Appl. 7 (1963) 2, 167-175.

[4] Griffiths, T. V.: The unsolvability of the equivalence problem for $A$-free nondeterministic generalized machines - J. ACM 15 (1968) 3, 409-413.

[5] Аrвiв, M. A.: Realization of stochastic systems - Ann. Math. Statistics 38 (1967) $223-233$.

[6] Nasu, M., Honda, N.: Mappings induced by PGSM-mappings and some recursively unsolvable problems of finite automata - Information and Control 15 (1969) 3, 250-273. 